\title{
Monte Carlo simulation of boson lattices
}

\author{
Vesa Apaja \\ Institut für Theoretische Physik, Johannes-Kepler Universität, A-4040 Linz, Austria \\ Olav F. Syljuåsen \\ Nordita, Blegdamsvej 17, DK-2100, Copenhagen Ø, Denmark
}

\begin{abstract}
Boson lattices are theoretically well described by the Hubbard model. The basic model and its variants can be effectively simulated using Monte Carlo techniques. We describe two newly developed approaches, the Stochastic Series Expansion (SSE) with directed loop updates and continuous-time Diffusion Monte Carlo (CTDMC). SSE is a formulation of the finite temperature partition function as a stochastic sampling over product terms. Directed loops is a general framework to implement this stochastic sampling in a non-local fashion while maintaining detailed balance. CTDMC is well suited to finding exact ground-state properties, applicable to any lattice model not suffering from the sign problem; for a lattice model the evolution of the wave function can be performed in continuous time without any time discretization error. Both the directed loop algorithm and the CTDMC are important recent advances in development of computational methods. Here we present results for a Hubbard model for anti-ferromagnetic spin-1 bosons in one dimensions, and show evidence for a dimerized ground state in the lowest Mott lobe.
\end{abstract}

Keywords: Optical lattices; Quantum Monte Carlo; Antiferromagnetic Boson systems; Dimerization

\section{INTRODUCTION}

An optical lattice can be made by applying orthogonal laser beams to an ultracold gas of atoms. As a result ${ }^{87} \mathrm{Rb}$ or ${ }^{23} \mathrm{Na}$ atoms can be trapped to form a perfect lattice. These atoms have a hyperfine spin 1 , with either a ferromagnetic $\left({ }^{87} \mathrm{Rb}\right)$ or antiferromagnetic $\left({ }^{23} \mathrm{Na}\right)$ interaction. Unpolarized ${ }^{23} \mathrm{Na}$ atoms have spin-correlated Mott insulating states 1 , and it has been suggested ${ }^{2}$ that the ground state is a dimer phase in one, two and three dimensions. An effective Hamiltonian of spin-1 bosons in an optical lattice has the Bose-Hubbard form, supplemented with a term that describes the spin interaction of atoms on the same lattice site ${ }^{3}$,

$$
H=-t \sum_{\langle i j\rangle, \alpha}\left(a_{i \alpha}^{\dagger} a_{j \alpha}+c c .\right)-\mu \sum_{i} \hat{n}_{i}+\frac{U}{2} \sum_{i} \hat{n}_{i}\left(\hat{n}_{i}-1\right)+\frac{U_{2}}{2} \sum_{i}\left(\vec{S}_{i}^{2}-2 \hat{n}_{i}\right),
$$

where the spin components $\alpha=x, y, z$ form a basis where the spin operator at site $i$ is written in terms of boson operators $a_{\alpha}^{\dagger}$ as $S_{i}^{\alpha}=-i \epsilon^{\alpha \beta \gamma} a_{\beta}^{\dagger} a_{\gamma}$. ( $\epsilon^{\alpha \beta \gamma}$ is the totally antisymmetric Levi-Civita tensor). In this basis the antiferromagnetic spin interaction has no sign problem. The value of $U_{2} / U$ can be determined from experimental scattering lengths.

$\mathrm{SSE}^{4}$ is an exact scheme, where the quantum partition function is expanded as a power series in a given basis $\{|s\rangle\}$,

$$
Z=\operatorname{Tr}\left\{e^{-\beta H}\right\}=\sum_{s} \sum_{n=0}^{\infty} \frac{-\beta^{n}}{n !}\left\langle s\left|H^{n}\right| s\right\rangle
$$

where $\beta=1 /\left(k_{B} T\right)$ is the inverse temperature. Typically the low-temperature phases we are simulating have at most 1-3 atoms per site, so even with the spin degrees of freedom the required number of single-site states is rather small. The Hamiltonian (1.1) couples at most two neighboring sites, so SSE splits the partition function to a sum of products of bond and site operations.

To generate the terms in SSE we employ directed loop updates ${ }^{5}$. The basic idea is to pick a few well chosen elementary update operations, that change the bond or site operators to each other. Each update affects only a single site (and operator) at a time and is applied in a loop among the operators in the product that is the current term of the SSE sum. The outcome is a new product of operators, and, thanks to the looping, this new product is also a term in the partition function. In addition one controls the number of operators in the product by adding or removing diagonal operators; they don't change the state so their addition or removal won't disrupt the continuity of the sequence. The rules for how the loop travels follow from the detailed balance condition, which is used to ascertain that the operators appear in the products with proper weights.

If the Hilbert space is finite one can formulate Diffusion Monte Carlo (DMC) in continuous time 6.7. DMC is a stochastic simulation of the imaginary-time evolution operator $e^{-H \tau}$. For an infinitesimal time step $d \tau$ this operator describes one out of three possible actions: 1) No action, the current state is unchanged, 2) Transition to a new state, 
and 3) The weight of the current state changes. Action 3) is needed due to the in general non-Markovian nature of the evolution operator. The probabilities for the different actions $p(i), i=1,2,3$ can be read directly off the Hamiltonian. The probability $p(2)$ is determined by off-diagonal elements of the Hamiltonian and is therefore of order $d \tau$. Also $p(3)$ is of order $d \tau$ because it describes the deviation from Markovian evolution, thus only $p(1)$ is of order unity. It follows that the DMC can be treated as a continuous-time simulation of a radioactive (multi-channel) decay problem, where decay-times are generated according to the exponential distribution: $\tau_{\text {decay }}=-\ln (r) d \tau /(1-p(1))$ where $r$ is a random number uniformly distributed between 0 and 1 . Having generated a decay-time the system is moved directly to the time of decay and the appropriate decay process is chosen dependent on the ratio $p(2) / p(3)$. CTDMC can be combined with other standard improvements of DMC such as importance sampling, reweighting and forward-walking 8 .

\section{RESULTS}

Fig. [1 shows the phase boundaries of the two lowest Mott insulating phases in the cases $U_{2} / U=0.2$ and $U_{2} / U=0.4$. Scattering between spin states stabilizes the second lobe, while the first lobe is slightly reduced in size.
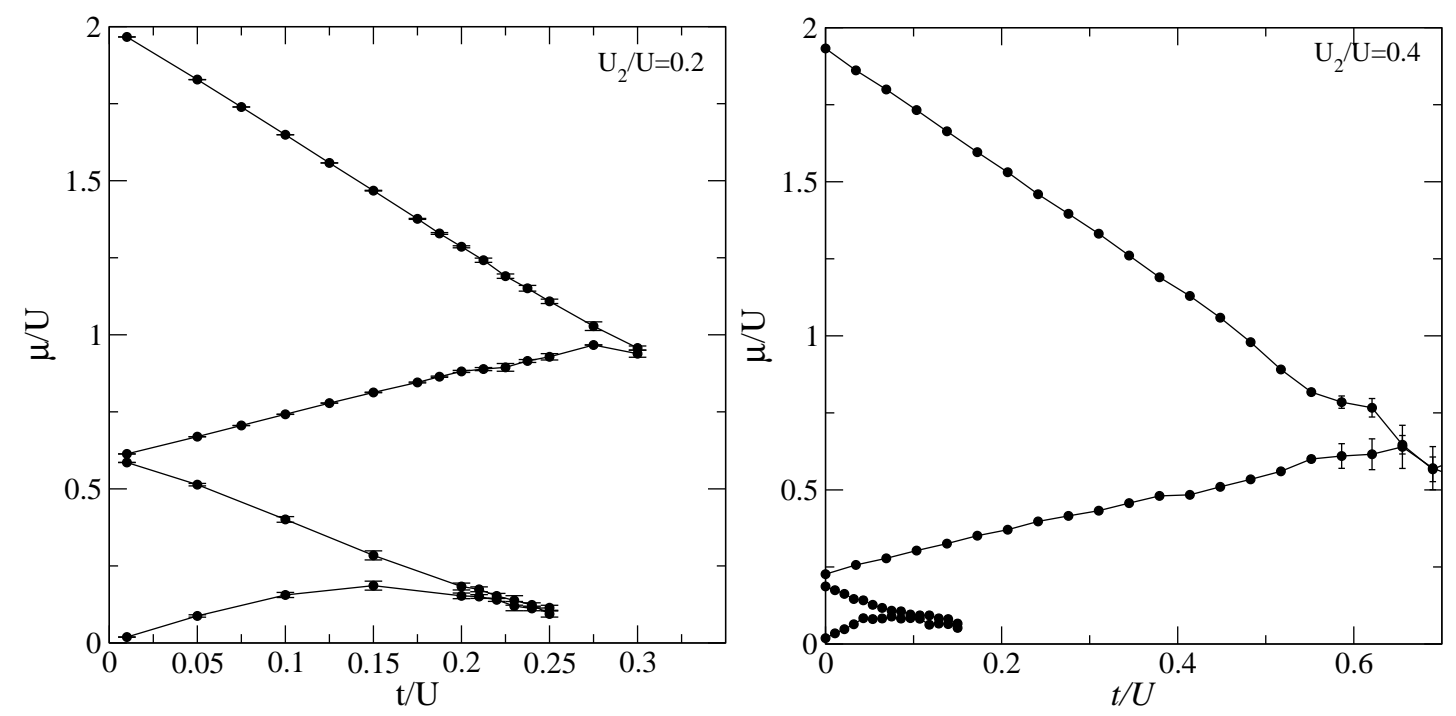

FIG. 1: The phase boundaries between Mott insulating and superfluid regions of a $1 \mathrm{D}$ chain for $U_{2} / U=0.2$ (left panel) and $U_{2} / U=0.4$ (right panel) at zero temperature. The results were obtained using CTDMC to compute ground state energies $E_{L}(N)$ for different particle numbers $N$ and system sizes $L$. The upper(lower) boundary $\mu_{p+}\left(\mu_{p-}\right)$ of the Mott lobe with $p$ bosons per site was obtained from the finite size values for $\mu_{p \pm}= \pm\left(E_{L}(p L \pm 1)-E_{L}(p L)\right)$ extrapolated to $L \rightarrow \infty$ using $L=8,16,20$ and 24 .

To study the structure of the Mott insulating phase with one atom per site we have measured the bond hopping or dimer susceptibility

$$
\mathcal{X}(q)=\frac{1}{t^{2} \beta N}\left(\left\langle N_{q} N_{-q}\right\rangle-\left\langle N_{q}\right\rangle\left\langle N_{-q}\right\rangle\right),
$$

where $N_{q}$ is the Fourier transform of $N_{i}$, the number of hopping operators on bond $i$. Because of the on-site spin scattering, the hopping operators tend to couple pairwise adjacent sites, indicated by a peak at wave number $q=\pi$. For a dimer state in an infinite system this peak height should diverge, and we demonstrate this in Fig. 2 For larger than 32 sites the computation of the susceptibility becomes exceedingly slow, as autocorrelation times increase rapidly. This is due to our non-optimal treatment of the terms of type $a_{x}^{\dagger} a_{x}^{\dagger} a_{y} a_{y}$ which changes two spin indices on the same site simultaneously. In order to build such terms out of loop changes where only one spin index changes we have included intermediate, auxiliary terms $a_{x}^{\dagger} a_{y}$ in the Hamiltonian. These auxiliary terms are generally present when the loop is being built, but we do not allow a loop to close if any of them are present.

The left panel of Fig. [3 show how the dimer state is suppressed as one moves from the insulating to the superfluid state. The hopping parameter $t$ is kept fixed and we move out of the insulating phase by increasing $\mu$. The corresponding density, as it increases from unity, is shown in the right panel of Fig. [3. 


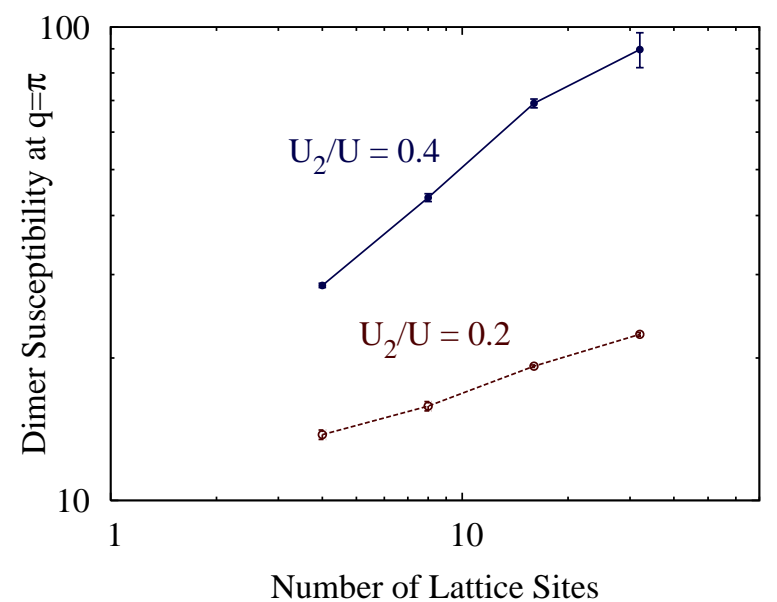

FIG. 2: Finite size scaling of the dimer susceptibility $\mathcal{X}(q)$ at $q=\pi$ in the Mott insulating phase of a $1 \mathrm{D}$ chain at $\beta=150$ for two values of $U_{2} / U$ indicated in the figure. We show the results for $L=4,8,16$ and 32 sites, those for $U_{2} / U=0.4$ were computed at $t=0.1, \mu=0.1$, and those for $U_{2} / U=0.2$ at $t=0.15, \mu=0.25$.
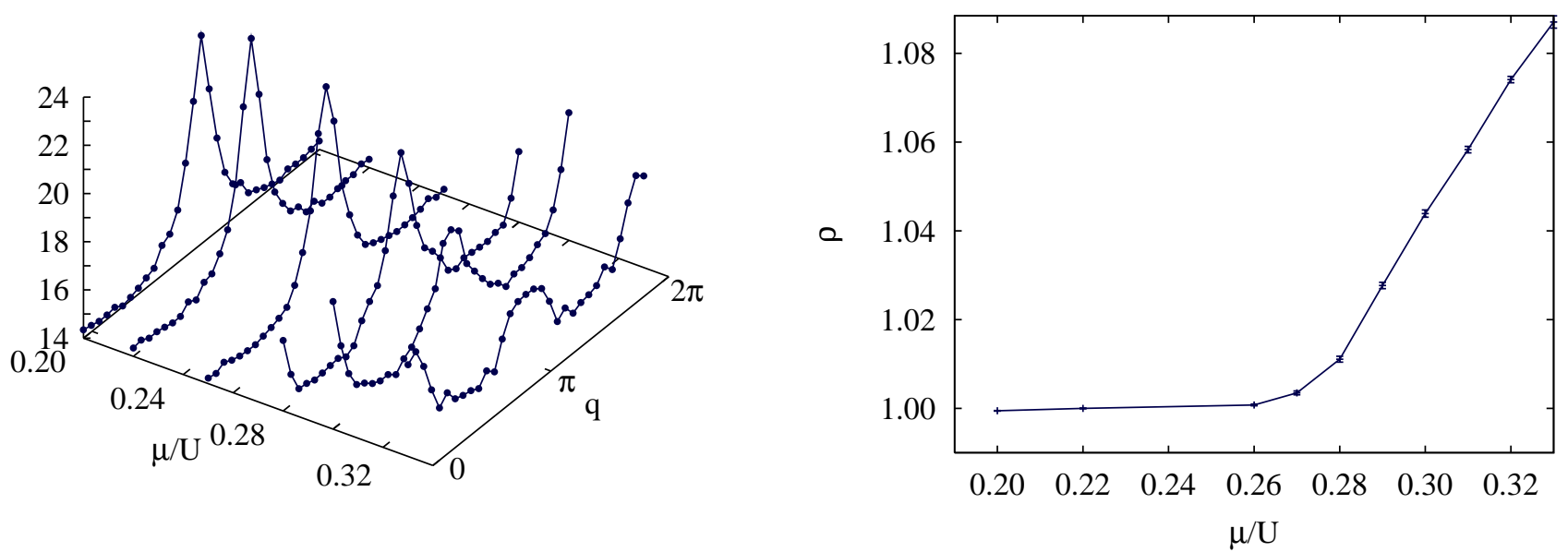

FIG. 3: The left panel shows the dimer susceptibility $\mathcal{X}(q)$ at the upper edge of the first Mott lobe (see the left panel of Fig. 1) of a $1 \mathrm{D}$ chain with 32 sites. The susceptibility is plotted as a function of the chemical potential $\mu / U$ and the wave number $q$. The data was computed at $t=0.15, \beta=150$ and $U_{2} / U=0.2$. The statistical error is less than 0.2 in the vertical scale. The right panel shows the corresponding density.

1 E. Demler and F. Zhou, Phys. Rev. Lett. 88, 163001 (2002).

2 S. K. Yip, Phys. Rev. Lett. 90, 250402 (2003).

3 A. Imambekov, M. Lukin, and E. Demler, Phys. Rev. Lett. 93, 120405 (2004).

4 A. W. Sandvik and J. Kurkijärvi, Phys. Rev. B 43, 5950 (1991).

5 O. F. Syljuåsen and A. W. Sandvik, Phys. Rev. E 66, 046701 (2002).

${ }^{6}$ E. Farhi and S. Gutmann, Ann. Phys. 213, 182 (1992).

7 O. F. Syljuåsen, Phys. Rev. B 71, 020401(R) (2005).

8 M. P. Nightingale and C. J. Umrigar, in Advances in Chemical Physics 105, eds. D. M. Ferguson et al. (John Wiley, NY, 1998), chapter 4. 